\title{
Erratum to: Onset-related differences in neural substrates of tinnitus-related distress: the anterior cingulate cortex in late-onset tinnitus, and the frontal cortex in early-onset tinnitus
}

\author{
Jae-Jin Song • Sven Vanneste $\cdot$ Winfried Schlee $\cdot$ \\ Paul Van de Heyning • Dirk De Ridder
}

Published online: 21 January 2014

(c) Springer-Verlag Berlin Heidelberg 2014

\section{Erratum to: Brain Struct Funct}

\section{DOI 10.1007/s00429-013-0648-x}

The affiliation of the corresponding author was incorrectly published in the original publication; the correct affiliation should read as given below.

Jae-Jin Song

Department of Otorhinolaryngology-Head and Neck Surgery, Seoul National University Bundang Hospital, 166 Gumi-Ro, Bundang-Gu, Gyeonggi-Do 463-707, Korea.

The online version of the original article can be found under doi:10.1007/s00429-013-0648-x.

J.-J. Song ( $ه)$

Department of Otorhinolaryngology-Head and Neck Surgery, Seoul National University Bundang Hospital, 166 Gumi-Ro, Bundang-Gu, Gyeonggi-Do 463-707, Korea

e-mail: jjsong96@gmail.com

S. Vanneste $\cdot$ P. Van de Heyning

School of Behavioral and Brain Sciences,

The University of Texas at Dallas, Richardson, USA

W. Schlee

Institute for Psychology and Education,

University of Ulm, Ulm, Germany

P. Van de Heyning

Department of Otorhinolaryngology and Head and Neck

Surgery, University Hospital Antwerp, Edegem, Belgium

D. De Ridder

Section of Neurosurgery, Department of Surgical Sciences,

Dunedin School of Medicine, University of Otago,

Dunedin, New Zealand 